\title{
Pretraumatic Stress Reactions in Soldiers Deployed to Afghanistan
}

Clinical Psychological Science 2015, Vol. 3(5) 663-674 (c) The Author(s) 2014 Reprints and permissions: sagepub.com/journalsPermissions.nav DOI: $10.1177 / 2167702614551766$ cpx.sagepub.com

(SAGE

\author{
Dorthe Berntsen $^{1}$ and David C. Rubin ${ }^{1,2}$ \\ ${ }^{1}$ Aarhus University and ${ }^{2}$ Duke University
}

\begin{abstract}
Posttraumatic stress disorder is a diagnosis related to the past. Pretraumatic stress reactions, as measured by intrusive involuntary images of possible future stressful events and their associated avoidance and increased arousal, have been overlooked in the PTSD literature. Here we introduce a scale that measures pretraumatic stress reactions providing a clear future-oriented parallel to the posttraumatic stress reactions described in the diagnostic criteria for PTSD. We apply this Pretraumatic Stress Reactions Checklist (PreCL) to Danish soldiers before, during, and after deployment to Afghanistan. The PreCL has good internal consistency and is highly correlated with a standard measure of PTSD symptoms. The PreCL as answered before the soldiers' deployment significantly predicted level of PTSD symptoms during and after their deployment, while controlling for baseline PTSD symptoms and combat exposure measured during and after deployment. The findings have implications for the conceptualization of PTSD, screening, and treatment.
\end{abstract}

\section{Keywords}

autobiographical memory, memory, posttraumatic stress disorder, trauma, war

Received 1/4/14; Revision accepted 6/21/14

Posttraumatic stress disorder (PTSD) is a diagnosis related to the past, as indicated by the prefix "post" as well as by the diagnostic requirements (American Psychiatric Association, 2013). Curing PTSD is healing the wounds of the past. Some years ago, however, an entertainment newspaper, the Onion, turned the notion of PTSD on its head by featuring a satirical article stating that an increasing number of U.S. soldiers suffered from pretraumatic stress ("Report: More U.S. Soldiers," 2006). This alleged syndrome involved vivid intrusive images and "flash-forwards" of stressful events that the soldiers might potentially experience or witness during their future deployment. The soldiers were suffering from possible future events, events that had not yet taken place. They were wounded, not by their past, but by their own projections of the future. Consistent with the ironic approach, the Onion article stated that a risk factor for this disorder was watching more than three hours of television news per day.

But are pretraumatic stress reactions fiction? From its introduction in 1980 until 2013, PTSD was classified as an anxiety disorder (American Psychiatric Association, 1980,
2000, 2013), and so concern for future negative events is to be expected (Ehlers \& Clark, 2000). Indeed, apprehension of catastrophic or socially embarrassing future events is often observed in anxiety disorders (e.g., Clark, 1999; Ottaviani \& Beck, 1987). Here we show that pretraumatic stress reactions, in terms of intrusive images and dreams about negative future events, accompanied by attempts at avoidance and increased levels of arousal, are a real aspect of the phenomenology of PTSD, which hitherto has been overlooked.

The term pretraumatic stress reactions here designates disturbing future-oriented cognitions and imaginations as measured in terms of a direct temporal reversal of the conceptualizations of past-directed cognitions in the PTSD diagnosis. For example, the concept of "repeated, disturbing memories, thoughts, or images of a stressful

\footnotetext{
Corresponding Author:

Dorthe Berntsen, Center on Autobiographical Memory Research, Department of Psychology and Behavioural Sciences, Aarhus University, Bartholins Allé 9, 8000 Aarhus C. Denmark

E-mail: dorthe@psy.au.dk
} 
experience from the past" is reformulated to the futuredirected version of having "repeated, disturbing imagined scenes, thoughts, or images concerning a possible stressful experience in the future." Having pretraumatic stress reactions, as defined here, does not imply that the person has had no prior traumatic events or that the person has no symptoms of PTSD in relation to such prior events. On the contrary, we propose that pretraumatic stress reactions are a central (but largely ignored) aspect of the phenomenology of PTSD.

We present data from Danish soldiers whose levels of post- and pretraumatic stress were measured before, during, and after deployment to Afghanistan. Because our data were collected before the introduction of the fifth edition of the Diagnostic and Statistical Manual of Mental Disorders (DSM-5), they are based on the PTSD diagnosis as described in DSM-IV (American Psychiatric Association, 2000). We demonstrate that pretraumatic stress reactions exist, and that pretraumatic stress reactions as measured before deployment are a reliable predictor of PTSD symptoms during and after deployment, even when controlling for baseline PTSD symptoms and combat exposure. Our findings have important implications for the conceptualization of PTSD, as well as for screening and treatment.

\section{Remembering the Past and Imagining the Future}

PTSD is conceived of as a disorder caused by past events. A person suffering from PTSD has repeated intrusive memories and dreams associated with the past traumatic event(s), persistent avoidance of potential reminders of the past traumatic event(s), negative alterations in cognition and mood associated with the past event(s), as well as alterations in arousal and reactivity that are also associated with the past traumatic event(s). All these symptoms are to have begun after the occurrence of the traumatic event(s) in question, according to the diagnostic criteria (American Psychiatric Association, 2013).

Consistent with this emphasis on the past, hundreds of studies have examined how PTSD impacts memory (for reviews and discussions, see Brewin, 2007; Brewin \& Holmes, 2003; Dalgleish, 2004; Kihlstrom, 2006; Klein, Caspi, \& Gil, 2003; McNally, 2003a, 2003b; Rubin, Berntsen, \& Bohni, 2006). At the same time, little is known as to how PTSD affects constructing possible events in the future. This oversight is surprising. First, anxiety disorders generally involve negative futureoriented cognition in terms of worries and spontaneously occurring images of threatening situations (see Clark, 1999, for a review). Thus, given the fact that PTSD traditionally has been viewed as an anxiety disorder, and thus should be concerned with the appraisal of imminent threat (Ehlers \& Clark, 2000), we would expect futuredirected cognitions to be common also in PTSD.

Second, a rapidly growing body of neurocognitive research has demonstrated that factors affecting memory for past events tend to exert similar effects on the construction of possible events in the future (for reviews, see D'Argembeau, 2012; Szpunar, 2010). Such studies have shown that the two processes recruit largely the same areas in the brain (e.g., Addis, Wong, \& Schacter, 2007; Botzung, Denkova, \& Manning, 2008; Okuda et al., 2003; Schacter et al., 2007; Szpunar, Watson, \& McDermott, 2007) and are similarly affected by a range of behavioral manipulations (e.g., D'Argembeau \& Van der Linden, 2004; Spreng \& Levine, 2006; Szpunar \& McDermott, 2008; but see Berntsen \& Bohn, 2010, and Rubin, 2014, for some deviations from this pattern). The ability to remember the personal past and imagine possible events in the personal future also seems to develop around the same time in the preschool years (e.g., Suddendorf, 2010), and shows similar effects of aging (e.g., Addis, Musicaro, Pan, \& Schacter, 2010; Addis, Wong, \& Schacter, 2008) as well as similar deficits in mental disorders, such as depression and schizophrenia (e.g., D'Argembeau, Raffard, \& Van der Linden, 2008; Williams et al., 1996). Extrapolating these findings to PTSD gives us reasons to assume that not just memories of the past but also simulations of future events would be affected by this disorder. We have been able to identify one study directly examining this possibility. Brown et al. (2013) asked 12 combat veterans diagnosed with PTSD and 16 veterans without PTSD to generate memories and simulate future events in response to neutral cue words. The veterans with PTSD reported more overgeneral events for both temporal directions. They also reported more events with a combat-related content, but this effect was strongest for the past.

Here we take the idea of a parallelism one important step further by asking, as did the Onion ("Report: More U.S. Soldiers," 2006) article, whether symptoms of PTSD related to the past-that is, the intrusive memories, the attempts at avoiding reminders, and the associated increased levels of arousal-are mirrored by similar symptoms related to the future. In other words, would people suffering from posttraumatic stress reactions experience intrusive involuntary future projections of traumatic and stressful events to a similar extent as they experience intrusive memories? Would they try to avoid cues to these images of future events to the same extent as they avoid cues to the memories, and would they show increased arousal in association with these reactions? On the basis of the parallels between remembering past events and imagining future events just reviewed, we predict that this indeed will be the case. Central to these predictions is also research on involuntary future 
projections, to which we now turn, showing that not only past events but also possible future events often come to mind involuntarily (unbidden).

\section{Involuntary Future Projections}

Involuntary autobiographical memories are memories of past events that come to mind spontaneously, that is with no preceding attempts at retrieval (Berntsen, 1996). Unlike intrusive trauma memories in PTSD, involuntary memories in daily life typically are about mundane and predominantly positive events. Still, they share a number of characteristics with intrusive memories in PTSD by being spontaneously (nonstrategically) retrieved, uncontrollable, typically cued by concrete perceptual features of the environment, dominated by visual imagery and by often having identifiable emotional impact at the time of retrieval (see Berntsen \& Watson, 2013, for a recent review) As a future parallel, Berntsen and Jacobsen (2008) introduced the notion of involuntary future event representations-defined as representations of possible personal future events that come to mind involuntarilythat is, with no preceding attempts at generating the imagined event. In a diary study involving a student sample, Berntsen and Jacobsen found that involuntary future events were facilitated by situational cues in much the same ways as involuntary memories, in that the cues were most frequently overlapping situational features in terms of objects, people, themes, or places. They also found that involuntary future projections differed from strategically generated future projections in ways that paralleled the differences observed between involuntary and voluntary memories, in that involuntary future projections were more specific, more vivid, and less emotionally positive and involved more negative mood impact as compared with their voluntary counterparts. These findings were replicated in samples of high versus low worriers (Finnbogadóttir \& Berntsen, 2011). Finnbogadóttir and Berntsen (2013) showed that involuntary future projections occurred as frequently as involuntary memories in daily life, with an average of about 20 involuntary future projections (or memories) per day.

Involuntary future projections are also found in clinical populations. They have been observed in both panic disorders and social anxiety disorders (see Clark, 1999, for a review). For example, Ottaviani and Beck (1987) described several cases with automatic and catastrophic imagery preceding or accompanying the panic attack, such as an attorney imaging himself fainting in the courtroom. More recently, involuntary future projections have been studied systematically in relation to depression and bipolar disorder (Deeprose \& Holmes, 2010; Deeprose, Malik, \& Holmes, 2011; Holmes, Crane, Fennell, \& Williams, 2007). Holmes et al. (2007) examined images of suicide in 15 depressed and previously suicidal patients. These images were vivid and persistent and could be conceived of as flash-forwards to the suicide. Deeprose and Holmes (2010) introduced the Impact of Future Events Scale (IFES) by adapting the Impact of Event Scale (Horowitz, Wilner, \& Alvarez, 1979) to be directed toward future instead of past events. Higher scores on the IFES were associated with higher levels of dysphoria (Deeprose \& Holmes, 2010) and bipolar disorder (Deeprose et al., 2011).

However, by asking broadly about images of the future (e.g., "Pictures about the future popped into my mind") the IFES does not differentiate between stressful intrusive involuntary future projections and mundane future projections, which are often positive. In fact, Deeprose et al. (2011) found that the emotional content of the future events was positive in two thirds of the cases, which is equivalent to the frequencies of positive versus negative future events found in diary studies of involuntary memories and future projections with nonclinical populations (e.g., Berntsen \& Jacobsen, 2008) and in studies of voluntary autobiographical memory more generally (Walker, Skowronski, \& Thompson, 2003). Thus, most of the future projections measured by the IFES may be adaptive simulations of mundane future events, even in clinical populations. Previous research therefore leaves a need for a measure of pretraumatic stress reactions that specifically captures emotionally negative and intrusive future projections, and their potentially associated reactions of avoidance and increased arousal.

\section{The Present Study}

The present study has three central aims. The first is to introduce a scale that measures pretraumatic stress reactions to future imagined events in a way that provides a clear future-oriented parallel to the emotionally negative stress reactions described in the diagnostic criteria for PTSD. The second aim is to examine its correlations with PTSD symptoms (i.e., symptoms related to stressful past events) within a population of Danish soldiers deployed to Afghanistan. The third aim is to use pretraumatic stress reactions measured before the soldiers' deployment to predict level of PTSD symptoms during and after their deployment, while controlling for PTSD symptoms (assessed before deployment) and combat exposure (measured during and after deployment).

Our measure of pretraumatic stress reactions is developed from the PTSD Checklist (PCL; Weathers, Litz, Huska, \& Keane, 1994), which measures the 17 official symptoms of PTSD in the DSM-IV (American Psychiatric Association, 2000). The first eight items concern reexperiencing and avoiding reminders of traumatic events in the past. We reformulated these eight items to address 
preexperiencing and avoiding reminders of possible traumatic events in the future, while leaving the remaining nine items unchanged. We designate our pretraumatic stress reaction measure the Pretraumatic Stress Reactions Checklist (PreCL).

We predict that pretraumatic stress reactions, as measured by the PreCL, form an independent aspect of the phenomenology of PTSD that shows high internal consistency and that is highly correlated with, but not identical to, the posttraumatic stress reactions measured by the standard PCL. Therefore we also expect the two scales to correlate at similar levels with known risk factors for the development of PTSD, including earlier traumas, depression, and neuroticism. Because they are temporally reversed, we expect the first eight items of the PreCL to have lower correlations with the corresponding eight items in the PCL than do the remaining nine items, which are identical in both the PCL and PreCL. We nonetheless predict high correlations between the two sets of items within each scale, thereby demonstrating high internal consistency for both the PreCL and the PCL. This prediction is based on our expectation that respondents will answer the last nine unchanged items differently in the two scales in the context of the differently worded instructions and the differently worded preceding eight items. We expect the PreCL obtained before deployment to predict PTSD symptoms during and after deployment, when controlling for baseline PTSD symptoms (measured before deployment) and combat exposure (measured during and after deployment). This is consistent with our assumption that pretraumatic stress reactions form an independent aspect of the phenomenology of PTSD.

\section{Method}

The present study was part of a larger data collection, which involved a number of psychometric measures related to PTSD symptoms, mental health, and well-being in a team of Danish soldiers before, during, and after a 6-month deployment to Afghanistan in 2009. A detailed analysis of trajectories for the development of PTSD symptoms across time, their risk factors, and attrition rates are available elsewhere (Berntsen et al., 2012). Here we focus on those parts of the data collection that are of theoretical relevance for the present work.

\section{Analysis sample}

The participants in the present study belonged to the Danish Contingent of the International Security Assistance Force 7 (ISAF 7). These soldiers were deployed for 6 months to the forward-operating bases in the Helmand province of southern Afghanistan, where the Danish soldiers took part in the British-led Task Force Helmand.
Their deployment involved substantial combat exposure and attacks by the enemy (Berntsen et al., 2012). At least $80 \%$ of the team reported that they had (a) experienced shootings near or against their camp, (b) passed through areas with combat, and (c) passed through areas with danger of roadside bombs. Around half of the soldiers were combat soldiers, about $70 \%$ of whom reported having killed an enemy. The analysis sample was 218 soldiers $(15$ females; mean age $=25.58$ years, range $18-51$ years) who provided answers to our measures of posttraumatic stress reactions for the four assessments included in the present study: before deployment (before), during deployment (during), 1 to 3 weeks after return from deployment (return), and 2 to 4 months after return (return +3 months).

Our analysis sample formed a subsample of the 610 soldiers who completed the questionnaire before deployment. To identify possible attrition biases, we compared the analysis sample $(n=218)$ with the remaining subsample $(n=392)$ on relevant background variables, including measures from before deployment of age, gender, time spent in the military, earlier traumas, depression, a range of psychological problems, personality traits, positive and negative affect, PTSD symptoms, pretraumatic stress reactions; measures from during deployment of combat exposure, PTSD symptoms, and pretraumatic stress reactions; measures at return from deployment of combat exposure and PTSD symptoms; and measures obtained 2 to 4 months after return of combat exposure, PTSD symptoms, and pretraumatic stress reactions. The analysis sample had fewer earlier traumatic events, $t(594)=2.37, p<.05$, scored slightly higher on a neuroticism, $t(594)=2.30, p<.05$, and scored lower on extraversion, $t(594)=3.41, p<.001$. No other significant differences were found.

\section{Materials}

The data collections included many different psychometric measures, of which the following were relevant to the present analyses (the PCL and the PreCL were never answered consecutively but always separated by several other questionnaires in the questionnaire package, which varied slightly across the assessment times).

PTSD Checklist (PCL). Posttraumatic Stress Disorder Checklist, Civilian Version (Blanchard, Jones-Alexander, Buckley, \& Forneris, 1996; Weathers et al., 1994) is a standardized checklist for PTSD symptoms, which has been used in previous studies of deployment-related PTSD. The scale consists of 17 items (equivalent to the 17 PTSD symptom categories in the DSM-IV), with a possible sum score ranging from 17 to 85 . The internal consistencies (Cronbach's $\alpha$ ) of the PCL in the present population of 
soldiers are .85 (Berntsen et al., 2012). The PCL was answered at all four assessment times.

Pretraumatic Stress Reactions Cbecklist (PreCL). This is a checklist for pretraumatic stress reactions developed for the purpose of the present study. The scale consists of 17 items (equivalent to the 17 PTSD symptom categories in the DSM-IV; American Psychiatric Association, 2000), with a possible sum score ranging from 17 to 85 . The first 8 items of the scale are a temporal reversal of the first eight items of the PCL, directed toward the future instead of the past. The remaining 9 items are formulated identically to the items in the PCL, but are answered with regard to possible negative future events, instead of negative past events. The instructions for the PreCL are a temporal reversal of the instructions for the PCL (see Table 1, which also includes the temporally reversed items needed for a version of the PreCL based on the DSM-5; American Psychiatric Association, 2000). The PreCL was answered three times: before, during, and 3 months after return from deployment (internal consistencies are reported in the results section).

Combat Exposure Scale. The Combat Exposure Scale (Keane et al., 1989) is a standard measure of exposure to combat, such as episodes with hostile fire, firing at the enemy, or fellow soldiers being injured or killed during combat. The scale consists of seven items. The internal consistencies (Cronbach's $\alpha$ ) of the Combat Exposure Scale in the present population of soldiers are .85 (Berntsen et al., 2012). The Combat Exposure Scale was answered three times: during, at return, and 3 months after return from deployment.

Beck Depression Inventory-II. The Beck Depression Inventory-II (BDI-II; Beck, Steer, \& Brown, 1996) measures level of depression. The inventory consists of 21 items, with a possible sum score ranging from 0 to 63 . The BDI-II was answered at all four measurement times. The internal consistencies (Cronbach's $\alpha$ ) of the BDI-II in the present population of soldiers are .85 (Berntsen et al., 2012). Measures obtained before deployment are relevant for the present analyses.

Neuroticism. The scale for neuroticism is one of the five personality factors measured by the NEO Five-Factor Inventory (Costa \& McCrae, 1989). The neuroticism factor consists of 12 items with a possible sum score ranging from 12 to 60. The scale was answered before and 3 months after deployment. The internal consistencies (Cronbach's $\alpha$ ) of the neuroticism scale in the present population of soldiers are .77 (Berntsen et al., 2012). Measures obtained before deployment are relevant for the present analyses.
Trauma Life Experience Questionnaire. The Trauma Life Experience Questionnaire (TLEQ; Kubany et al., 2000) was here accommodated to a Danish population of soldiers. Here it consists of 20 questions addressing different types of traumas. The questionnaire measures how often (from never to more than five times) different traumas have been experienced. The internal consistencies (Cronbach's $\alpha$ ) of the TLEQ in the present population of soldiers are .70 (Berntsen et al., 2012). This scale was answered before deployment.

\section{Procedure}

Data for the assessment before deployment were collected by military psychologists 5 to 6 weeks before deployment during group sessions at a military camp in Denmark. The soldiers were informed about the study, including the fact that their responses were anonymous, would not be accessed by their leaders in the military, and would be used for research purposes only. Data for the assessment during deployment were collected in Afghanistan. All respondents had been deployed for at least 2 and less than 5 months, when they answered this set of questionnaires. The questionnaires were handed out by military personnel at Camp Bastion, Kabul International Airport, or Kandahar Airfield before the soldiers went home on leave. The soldiers submitted their answers in closed envelopes in secured (locked) mailboxes. The locked mailboxes were transported to the research team in Denmark. The data for the Return assessment were collected at a standard homecoming meeting organized by military psychologists and physicians. This data collection took place 1 to 3 weeks after the soldiers had returned from deployment. The data for the return +3 months assessment were collected at different military camps in Denmark, or through mail for those who had returned to civilian life, roughly 2 to 4 months after return from deployment (for more details on the study, see Berntsen et al., 2012).

\section{Results}

We first examine the internal consistency of the PreCL in comparison with the PCL, their means, and their correlations. We next examine the extent to which the PreCL, answered before deployment, predicts PTSD symptoms during and after deployment, when controlling for baseline PCL and combat exposure.

\section{Internal consistency, means and correlations}

The internal consistencies of the PreCL and PCL were similar. For the three measurement times at which both were 
Table 1. The Pretraumatic Stress Reactions Checklist (PreCL), Temporally Reversed Items

\section{DSM-IV Version}

Instructions: Below is a list of problems and complaints that people sometimes have before possible stressful life experiences in the future. Please read each one carefully, then circle one of the numbers to the right to indicate how much you have been bothered by that problem in the past month.

Item Temporally reversed formulation

1. Repeated, disturbing imagined scenes, thoughts, or images concerning a possible stressful experience in the future?

2. Repeated, disturbing dreams concerning a possible stressful experience in the future?

3. Suddenly acting or feeling as if a possible stressful experience in the future already were happening (as if you were pre-living it)?

4. $\quad$ Feeling very upset when something reminded you of a possible stressful experience in the future?

5. Having physical reactions (e.g., heart pounding, trouble breathing, sweating) when something reminded you of a possible stressful experience in the future?

6. Avoiding thinking about or talking about a possible stressful experience in the future or avoiding having feelings related to it?

7. Avoiding activities or situations because they reminded you of a possible stressful experience in the future?

8. Trouble imagining important parts of a possible stressful experience in the future?

\section{DSM-5 Version}

Instructions: Below is a list of problems and complaints that people sometimes have before a possible, very stressful experience in the future. Please read each one carefully, then circle one of the numbers to the right to indicate how much you have been bothered by that problem in the past month.

\begin{tabular}{ll} 
Item & Temporally reversed formulation \\
\hline 1. & Repeated, disturbing and unwanted images of a possible future stressful experience? \\
2. & Repeated, disturbing dreams of a possible future stressful experience? \\
3. & Suddenly acting or feeling as if a possible future stressful experience already were happening (as if you were pre- \\
living it)? & Feeling very upset when something reminded you of a possible future stressful experience? \\
5. & Having strong physical reactions (e.g., heart pounding, trouble breathing, sweating) when something reminded you \\
6. & Avoiding imaginings, thoughts or feelings related to a possible future stressful experience? \\
7. & activities, objects or situations)? \\
8. & Trouble imagining important parts of a possible future stressful experience? \\
10. & Blaming yourself or someone else for a possible future stressful experience or what has led up to it?
\end{tabular}

Note: The $D S M-I V$ version is rated on a scale from 1 to 5 ; the $D S M-5$ version is rated on a scale from 0 to 4 ; anchor points for both are not at all, a little bit, moderately, quite a bit, and extremely. Both are based on the relevant PCL (Weathers et al., 2013; Weathers, Litz, Huska, \& Keane, 1994). Both should be administered with the temporally nonreversed items included. The full scales may be obtained from the authors. ${ }^{1}$

completed, Cronbach's alpha was $.85, .86$, and .93 , for the PreCL and $.89, .87$, and .92 for the PCL ( $n s=213-218$ ).

The means and standard deviations for the two scales over the same three assessment times and results from repeated measures analysis of variance (ANOVA) are presented in Table 2. Both the PreCl and the PCL varied across the three assessments. The PreCL and the PCL were significantly different only at return +3 months, $t(214)=3.91, p<.001$, causing a significant interaction between assessment time and type of scale, $F(2,420)=$ 3.81, $p<.05 ; \eta_{p}{ }^{2}=.02$.

Examining just the first eight items that are temporally reversed in the PreCL yielded a similar pattern of findings (see Table 2). The sum scores for Items 1 to 8 of both scales varied across assessment times. Again, they differed significantly only at return +3 months, $t(215)=$ 2.35, $p<.001$, causing a significant interaction between assessment time and type of scale, $F(2,422)=5.23$, $p<.01 \eta_{p}^{2}=.03$.

Table 3 shows the correlations between the two scales at the three assessment times, when both scales were answered. As predicted, these correlations are high and less so for the temporally reversed items in the PreCL (Items 1 to 8) than for the items for which only the temporal context of the instructions differed between the two scales (Items 9 to 17).

The correlations between sums of Items 1 to 8 and 9 to 17 show that within each scale, the two sets of items 
Table 2. The PreCL and PCL Scores Before, During, and Three Months After Return From Deployment (Total and Items 1 to 8 Only)

\begin{tabular}{|c|c|c|c|c|c|}
\hline \multirow[b]{2}{*}{ Scale } & \multirow{2}{*}{$\frac{\text { Before }}{M(S D)}$} & \multirow{2}{*}{$\frac{\text { During }}{M(S D)}$} & \multirow{2}{*}{$\frac{\text { Return }+3 \text { months }}{M(S D)}$} & \multicolumn{2}{|c|}{ Effect of time } \\
\hline & & & & $F$ & $\eta_{p}^{2}$ \\
\hline \multicolumn{6}{|l|}{ Total items } \\
\hline PreCL $(n=211)$ & $22.85(6.81)$ & $20.80(5.79)$ & $21.00(7.39)$ & $11.93^{* * * * *}$ & .05 \\
\hline PCL $(n=218)$ & $22.82(7.68)$ & $21.19(6.20)$ & $21.96(7.62)$ & $6.07^{* * *}$ & .03 \\
\hline \multicolumn{6}{|l|}{ Items 1 to 8 only } \\
\hline PreCL $(n=212)$ & $10.44(3.33)$ & $9.23(2.61)$ & $9.26(3.44)$ & $16.79^{* * * * *}$ & .07 \\
\hline PCL $(n=218)$ & $9.99(3.64)$ & $9.30(2.80)$ & $9.62(3.28)$ & $3.97^{*}$ & .02 \\
\hline
\end{tabular}

Note: PCL $=$ PTSD Checklist; PreCL $=$ Pretraumatic Stress Reactions Checklist. Items 1 to 8 are temporally reversed in the PreCL. ${ }^{*} p<.05 .{ }^{* *} p<.001 . * * * k<<.0001$.

are equally highly correlated. In other words, temporally reversing Items 1 to 8 did not reduce the internal consistency of the PreCL relative to the PCL, lending further support to the observation that the internal consistency of the PreCL is as high as that of the PCL.

Finally, the two scales correlate similarly with measures of previous traumatic events, depression, and neuroticism (see Table 3). As expected, both scales are moderately to highly correlated with these well-known risk factors for anxiety disorders. The correlations are highest before deployment where the measures are obtained concurrently. The PreCL does not correlate more highly with these measures than the standard PTSD scale in any of the reported cases in Table 3. Rather, in cases where numerical differences are seen, the correlation coefficients are higher for the PCL. This eliminates the possibility that the PreCL simply is a proxy for such well-known vulnerability factors.

\section{PreCL as a predictor for PTSD symptoms}

Table 4 shows the results from hierarchical multiple regression analyses predicting level of PTSD symptoms during deployment, at return, and at 3 months after return, as three separate analyses. The top panel of Table

Table 3. Correlations Between the PreCL and PCL, Between Subsets of Items Within Each Scale, and With Measures of Earlier Traumatic Events, Depression, and Neuroticism

\begin{tabular}{|c|c|c|c|}
\hline \multirow[b]{3}{*}{ Correlation } & \multicolumn{3}{|c|}{ Measurement times before, during, and after deployment } \\
\hline & Before & During & Return +3 months \\
\hline & $r$ & $r$ & $r$ \\
\hline \multicolumn{4}{|l|}{ Between PreCL and PCL } \\
\hline Sums of Items 1 to 8 & .56 & .60 & .77 \\
\hline Sums of Items 9 to 17 & .85 & .82 & .90 \\
\hline Sums of all items & .79 & .79 & .89 \\
\hline \multicolumn{4}{|c|}{ Between sums for Items 1 to 8 and 9 to 17} \\
\hline Within PreCL & .62 & .57 & .70 \\
\hline Within PCL & .60 & .59 & .72 \\
\hline \multicolumn{4}{|c|}{ PreCL and TLEQ, BDI-II, and neuroticism } \\
\hline TLEQ (before) & .36 & .35 & .29 \\
\hline BDI-II (before) & .74 & .40 & .33 \\
\hline Neuroticism (before) & .45 & .34 & .26 \\
\hline \multicolumn{4}{|c|}{ PCL and TLEQ, BDI-II, and neuroticism } \\
\hline TLEQ (before) & .43 & .39 & .30 \\
\hline BDI-II (before) & .76 & .51 & .36 \\
\hline Neuroticism (before) & .49 & .40 & .30 \\
\hline
\end{tabular}

Note: BDI-II = Beck Depression Inventory; PCL $=$ PTSD Checklist; PreCL $=$ Pretraumatic Stress Reactions Checklist; TLEQ = Traumatic Life Event Questionnaire. $n s=203-218$. For the correlations, all $p s<.0005$. Items 1 to 8 are temporally reversed in the PreCL. 
Table 4. Hierarchical Multiple Regression Analyses Predicting PTSD Symptoms During Deployment, at Return, and Three Months After Return

\begin{tabular}{|c|c|c|c|c|c|c|}
\hline \multirow[b]{3}{*}{ Entire scale } & \multicolumn{6}{|c|}{ Measurement times during and after deployment } \\
\hline & \multicolumn{2}{|c|}{ During } & \multicolumn{2}{|c|}{ Return } & \multicolumn{2}{|c|}{ Return +3 months } \\
\hline & $\Delta R^{2}$ & $\beta$ & $\Delta R^{2}$ & $\beta$ & $\Delta R^{2}$ & $\beta$ \\
\hline Step 1 & $.50^{* * * * *}$ & & $.28 * * * *$ & & $.21^{* * * * *}$ & \\
\hline PCL before & & $.66^{* * * * *}$ & & $.46^{* * * *}$ & & $.45^{* * * *}$ \\
\hline Combat exposure $^{a}$ & & $.18^{* * *}$ & & $.22 * *$ & & .06 \\
\hline Step 2 & $.02 *$ & & $.07^{* * * *}$ & & $.05^{* * * * *}$ & \\
\hline PCL before & & .50 ***** & & .11 & & .15 \\
\hline Combat exposure & & $.16^{* *}$ & & $.20^{* * *}$ & & .01 \\
\hline PreCL before & & $.20^{*}$ & & $.44^{* * * *}$ & & $.38^{* * * * *}$ \\
\hline Total $R^{2}$ & $.52^{* * * * *}$ & & $.35^{* * * * *}$ & & $.26^{\text {***** }}$ & \\
\hline$N$ & 193 & & 197 & & 203 & \\
\hline Sums for Items 1 to 8 & $\Delta R^{2}$ & $\beta$ & $\Delta R^{2}$ & $\beta$ & $\Delta R^{2}$ & $\beta$ \\
\hline Step 1 & $.34 * * * *$ & & $.14^{* * * * *}$ & & $.18^{* * * * *}$ & \\
\hline PCL before & & $.52^{* * * *}$ & & $.26^{* * * * * *}$ & & $.41^{* * * * *}$ \\
\hline Combat exposure & & $.22^{* * * * *}$ & & $.24^{* * *}$ & & .07 \\
\hline Step 2 & $.04 * * * *$ & & $.08^{* * * * *}$ & & $.10 * * *$ & \\
\hline PCL before & & $.38^{* * * *}$ & & .07 & & $.20 * *$ \\
\hline Combat exposure & & $.19^{* * *}$ & & $.22^{* * *}$ & & .01 \\
\hline PreCL before & & $.25^{\text {***** }}$ & & $.34^{\text {***:*** }}$ & & $.39^{* * * * *}$ \\
\hline Total $R^{2}$ & $.38^{* * * *}$ & & $.22^{* * * *}$ & & $.28^{* * * * *}$ & \\
\hline$N$ & 193 & & 197 & & 203 & \\
\hline
\end{tabular}

Note: PCL $=$ PTSD Checklist; PreCL $=$ Pretraumatic Stress Reactions Checklist. Items 1 to 8 are temporally reversed in PreCL

${ }^{a}$ Combat exposure is always measured concurrently with the dependent variable.

${ }^{*} p<.05 .{ }^{* *} p<.01 .{ }^{* * * *} p<.0001$.

4 illustrates the outcomes of these analyses when the PCL score from before deployment and the concurrent measure of combat exposure were entered in the first step and the PreCL was entered in the second step. In all three analyses, the second step significantly increased the level of variance explained.

As also illustrated by Table 4, the PCL was not a significant predictor in two of the three analyses, after the PreCL had entered the regression equation. Accordingly, if the PreCL was entered in the first step and the PCL in the second step of these analyses, then the second step added significantly to the variance explained in only one of the three analyses-the one predicting PTSD symptoms during deployment $\left(\Delta R^{2}=.09\right)$. Taken together these findings suggest that PreCL completed before deployment predicts level of PTSD symptoms during and after deployment over and above the PCL completed before deployment and combat exposure as assessed concurrently with the dependent variable.

This pattern of results did not change if the sum scores for the TLEQ, BDI-II, and neuroticism were entered in the first step of the regression analyses, together with the
PCL and the measure of combat exposure. The PreCL as entered in Step 2 still added significantly to the amount of variance explained in all three analyses (with $\Delta R^{2}=$ $.02, .05$, and .03 , respectively, all $p s<.05$ ). In short, the PreCL answered before deployment explained independent variance in the level of PTSD symptoms at all three assessment times.

The PCL and PreCL as measured before deployment were highly correlated (Table 3 ), which might suggest a risk of multicollinearity affecting the results of the regression analyses. We therefore conducted the same analyses as the ones described in the top panel of Table 4, predicting the whole PCL, but this time we included as predictors only the sum scores for Items 1 to 8 of the PreCL and PCL, respectively. Thus, as predictor variables, we limited both scales to the sums of the items that were temporally reversed in the PreCL because they correlated only moderately $(r=.56)$ before deployment (see Table 3$)$. These analyses (presented in the bottom panel of Table 4) largely replicated the analyses based on the entire scales: When the sum score for the PreCL (Items 1 to 8) was entered in the second step, it significantly increased the 
amount of variance explained for all three analyses, with $\Delta R^{2}=.04, .08$, and .10, respectively. After the PreCL had entered, the sum score for the PCL (Items 1 to 8 ) remained significant in two of the three analyses, but not in the one predicting PTSD symptoms as measured at return (see Table 4). Accordingly, if the PreCL (Items 1 to 8) was entered in the first step and the PCL (Items 1 to 8 ) in the second step of these analyses, then the second step added significantly to the variance explained in only two of the three analyses: the ones predicting PTSD symptoms during deployment $\left(\Delta R^{2}=.10\right)$ and 3 months after return $\left(\Delta R^{2}=.03\right)$ but not at return $\left(\Delta R^{2}=.003\right)$. In sum, the PreCL (as answered before deployment) predicted PTSD symptoms during, at return, and 3 months after return over and above the PCL (answered before deployment) and combat exposure (measured concurrently with the dependent variable in the analyses). Taken together, these findings support the idea that pretraumatic stress reactions as measured by the PreCL form an independent aspect of the phenomenology of PTSD.

\section{Discussion}

We have introduced a scale for pretraumatic stress reactions-that is, disturbing future oriented cognitions and images measured in terms of a temporal reversal of the standard conceptualization of past directed cognition in PTSD. We have administered this scale in a group of soldiers before, during, and after deployment to Afghanistan. The study has two major findings. First, we have demonstrated that pretraumatic stress reactions are an important aspect of the phenomenology of PTSD: Involuntary intrusive images and thoughts of possible future events, their associated attempts at avoidance, and increased levels of arousal were experienced at the same level as posttraumatic stress reactions to past events before and during deployment. The two measures were highly correlated at all assessment times, even when the analyses were limited to the temporally reversed items (Table 3). They also correlated similarly with well-known predisposing factors for the development of PTSD. These findings suggest that pretraumatic and posttraumatic stress reactions are two subjectively different manifestations of the same underlying phenomenon. Second, we have demonstrated that pretraumatic stress reactions are a risk factor for the development of PTSD symptoms in that the PreCL completed before deployment predicted subsequent PTSD symptoms over and above baseline PTSD symptoms and combat exposure. These findings have several theoretical and practical implications.

First, the findings challenge the current understanding of PTSD as a disorder primarily related to the past. Instead, they are consistent with a new line of clinical research emphasizing the importance of mental imagery across a range of clinical disorders (for a review, see Pearson, Deeprose, Wallace-Hadrill, Heyes, \& Holmes, 2013) as well as with a rapidly growing literature demonstrating that episodic remembering and future simulations draw on overlapping neural and cognitive structures (e.g., D'Argembeau, 2012; Rubin, 2014; Schacter \& Addis, 2007; Szpunar, 2010). Following this evidence and given the fact that PTSD involves a well-documented bias toward threatening information (e.g., Bryant \& Harvey, 1995) and most likely a subjective sense of continuous threat (Ehlers \& Clark, 2000), we would expect not just memories for past events, but also simulations of future events, to be affected by the disorder. This is consistent with the present findings.

Second, the present findings hold potential implications for treatment. Currently, the most commonly used treatments for PTSD focus on the past traumatic events as they are represented in memory and appraised by the person. One common strategy is exposure therapy, including imaginal exposure, where the event is reexperienced in memory, and in vivo exposure, where the person is exposed to situations that involve reminders of the trauma and therefore are associated with avoidance. Although there is evidence for the beneficial effects of exposure therapies in their current memory-directed forms (Institute of Medicine, 2008), the present findings suggest that such therapies could be further advanced by including imaginal exposure to frequently intruding representations of stressful future events, maybe in combination with other forms of therapy, such as imagery rescripting (e.g., Arntz, 2012) or eye movement desensitization and reprocessing (Engelhard, van den Hout, Janssen, \& van der Beek, 2010) directed toward such event representations. Also, recent evidence that mindfulness-based exercises normalize cortisol levels and reduce PTSD symptoms among nurses (Kim et al., 2013) can be seen as consistent with the present findings, in that mindfulness therapies are not directed toward specific types of mental contents, but are likely to apply equally well to intrusive images of future and past events.

Third, our measure of pretraumatic stress reactions might serve as a useful instrument for screening for soldiers at risk for developing PTSD symptoms during deployment given the finding that pretraumatic stress reactions measured before deployment significantly predicted PTSD symptoms during and after deployment, even when controlling for baseline PTSD symptoms measured before deployment, combat exposure measured during and after deployment, as well as earlier traumas, depression, and neuroticisms measured before deployment. Future research should examine whether it also may be used as a screening instrument in relation to nonmilitary traumatic events as well as other subjectively stressful events, such as exams, medical procedures, or childbirth. 
More broadly, our findings suggest that intrusive images and dreams of future events and associated avoidance and increased arousal are experienced to the same extent as reexperiencing, avoidance, and increased arousal associated with past events. Therefore, assessing only the latter in diagnostic interviews and self-report questionnaires entails a one-sided focus on the past aspect of a phenomenology that includes both a future and a past temporal dimension.

The present study should be evaluated with a number of limitations in mind. Our sample included almost no females and very few participants older than 40. In addition, the soldiers generally presented with low levels of PTSD symptoms. Future research should examine whether the present findings can be generalized to more genderand age balanced populations, as well as populations with higher PTSD levels. Also, the present study did not examine the content or qualities of the soldiers' intrusive memories and future projections. It therefore leaves unanswered whether the future projections were vivid, whether the content of the future projections was thematically related to the content of the memories, and whether one of the two types of mental contents was experienced as being more emotionally intense and self-relevant than the other. Following previous work involving healthy populations, we would expect intrusive images related to the future to be seen as more self-relevant (e.g., Berntsen \& Jacobsen, 2008) and experienced as more emotionally extreme and stressful (Rubin, 2014) than intrusions of traumatic events in the past. Whether this indeed is the case in populations with reactions to severe traumatic events is a question for future research.

In summary, the present work opens an important new line of research on PTSD by showing that pretraumatic stress reactions are a genuine aspect of the phenomenology of this disorder. This discovery challenges the current understanding of the symptomatology of PTSD and calls for a reconsideration of procedures in relation to screening and treatment.

\section{Author Contributions}

The data collection for the present study was part of a more extensive data collection, which D. Berntsen designed and conducted in collaboration with the Danish military and members of her lab. D. Berntsen is also responsible for the formulation of the PreCL and its inclusion in the data collection. D. Berntsen and D. C. Rubin together designed the specific analyses for the present article and together interpreted and discussed the results. Both authors were actively involved in the writing process, for which D. Berntsen had a leading role.

\section{Acknowledgments}

We thank Yvonne Duval Thomsen, Kim Berg Johannessen, Robert Jonasen, Per Bach, Christoffer Holmgård Pedersen, and
Mette Bertelsen for their assistance, and Christin Ogle and Kaitlyn Batt for comments.

\section{Declaration of Conflicting Interests}

The authors declared that they had no conflicts of interest with respect to their authorship or the publication of this article.

\section{Funding}

We thank the Danish National Research Foundation Grant DNRF93, Danish Council for Independent Research: Humanities, Royal Danish Defense College, and NIH Grant R01MH066079 for support. The content is solely the responsibility of the authors and does not necessarily reflect the official views of the National Institutes of Health, or of the other agencies that have provided funding.

\section{Note}

1. The copyright for the scales is held by the authors (C2014, Berntsen \& Rubin). Permission is given to use the scales for research purposes.

\section{References}

Addis, D. R., Musicaro, R., Pan, L., \& Schacter, D. L. (2010). Episodic simulation of past and future events in older adults: Evidence from an experimental recombination task. Psychology and Aging, 25, 369-376.

Addis, D. R., Wong, A. T., \& Schacter, D. L. (2007). Remembering the past and imagining the future: Common and distinct neural substrates during event construction and elaboration. Neuropsychologia, 45, 1363-1377.

Addis, D. R., Wong, A. T., \& Schacter, D. L. (2008). Agerelated changes in the episodic simulation of future events. Psychological Science, 19, 33-41.

American Psychiatric Association. (1980). Diagnostic and statistical manual of mental disorders (3rd ed.). Washington, DC: Author.

American Psychiatric Association. (2000). Diagnostic and statistical manual of mental disorders (4th ed., text rev.). Washington, DC: Author.

American Psychiatric Association. (2013). Diagnostic and statistical manual of mental disorders (5th ed.). Washington, DC: Author.

Arntz, A. (2012). Imagery rescripting as a therapeutic technique: Review of clinical trials, basic studies, and research agenda. Journal of Experimental Psychopathology, 3, 121-126.

Beck, A. T., Steer, R. A., \& Brown, G. K. (1996). Beck Depression Inventory manual (2nd ed.). San Antonio, TX: Psychological Corporation.

Berntsen, D. (1996). Involuntary autobiographical memories. Applied Cognitive Psychology, 10, 435-454

Berntsen, D., \& Bohn, A. (2010). Remembering and forecasting. The relation between autobiographical memory and episodic future thinking. Memory \& Cognition, 38, 265-278.

Berntsen, D., \& Jacobsen, A. S. (2008). Involuntary (spontaneous) mental time travel into the past and future. Consciousness and Cognition, 17, 1093-1104. 
Berntsen, D., Johannessen, K. B., Thomsen, Y. D., Bertelsen, M., Hoyle, R. H., \& Rubin, D. C. (2012). Peace and war: Trajectories of posttraumatic stress disorder symptoms before, during and after military deployment in Afghanistan. Psychological Science, 23, 1557-1565.

Berntsen, D., \& Watson, A. L. (2013). Involuntary autobiographical memories in daily life and in clinical disorders. In T. Perfect \& S. Lindsay (Eds.), The Sage handbook of applied memory (pp. 501-519). Thousand Oaks, CA: Sage.

Blanchard, E. B., Jones-Alexander, J., Buckley, T. C., \& Forneris, C. A. (1996). Psychometric properties of the PTSD Checklist (PCL). Behaviour Research and Therapy, 34, 669-673.

Botzung, A., Denkova, E., \& Manning, L. (2008). Experiencing past and future personal events: Functional neuroimaging evidence on the neural bases of mental time travel. Brain and Cognition, 66, 202-212.

Brewin, C. R. (2007). Autobiographical memory for trauma: Update on four controversies. Memory, 15, 227-248.

Brewin, C. R., \& Holmes, E. A. (2003). Psychological theories of posttraumatic stress disorder. Clinical Psychology Review, 23, 339-376.

Brown, A. D., Root, J. C., Romano, T. A., Chang, L. J., Bryant, R. A., \& Hirst, W. (2013). Overgeneralized autobiographical memory and future thinking in combat veterans with posttraumatic stress disorder. Journal of Behavior Therapy and Experimental Psychiatry, 44, 129-134.

Bryant, R. A., \& Harvey, A. G. (1995). Processing threatening information in posttraumatic stress disorder. Journal of Abnormal Psychology, 104, 537-541.

Clark, D. M. (1999). Anxiety disorders: Why they persist and how to treat them. Behaviour Research and Therapy, 37, S5-S27.

Costa, P. T., \& McCrae, R. R. (1989). The NEO-PI/NEO-FFI manual supplement. Odessa, FL: Psychological Assessment Resources.

Dalgleish, T. (2004). Cognitive approaches to posttraumatic stress disorder: The evolution of multirepresentational theorizing. Psychological Bulletin, 130, 228-260.

D'Argembeau, A. (2012). Autobiographical memory and future thinking. In D. Berntsen \& D. C. Rubin (Eds.), Understanding autobiographical memory: Theories and approaches (pp. 311-330). Cambridge, England: Cambridge University Press.

D'Argembeau, A., Raffard, S., \& Van der Linden, M. (2008). Remembering the past and imagining the future in schizophrenia. Journal of Abnormal Psychology, 117, 247-251.

D'Argembeau, A., \& Van der Linden, M. (2004). Phenomenal characteristics associated with projecting oneself back into the past and forward into the future: Influence of valence and temporal distance. Consciousness and Cognition, 13, 844-858.

Deeprose, C., \& Holmes, E. A. (2010). An exploration of prospective imagery: The Impact of Future Events Scale. Behavioural and Cognitive Psychotherapy, 38, 201-209.

Deeprose, C., Malik, A., \& Holmes, E. A. (2011). Measuring intrusive prospective imagery using the Impact of Future Events Scale: Psychometric properties and relation to risk for bipolar disorder. International Journal of Cognitive Therapy, 4, 187-196.
Ehlers, A., \& Clark, D. M. (2000). A cognitive model of posttraumatic stress disorder. Behaviour Research and Therapy, 38, 319-345.

Engelhard, I. M., van den Hout, M. A., Janssen, W. C., \& van der Beek, J. (2010). Eye movements reduce vividness and emotionality of "flashforwards." Behavior Research and Therapy, 48, 442-447.

Finnbogadóttir, H., \& Berntsen, D. (2011). Involuntary mental time travel in high and low worriers. Memory, 19, 625-640.

Finnbogadóttir, H., \& Berntsen, D. (2013). Involuntary future projections are as frequent as involuntary memories, but more positive. Consciousness and Cognition, 22, 272-280.

Holmes, E. A., Crane, C., Fennell, M. J. V., \& Williams, J. M. G. (2007). Imagery about suicide in depression-"Flashforwards"? Journal of Behavior Therapy and Experimental Psychiatry, 38, 423-434.

Horowitz, M. J., Wilner, N., \& Alvarez, W. (1979). Impact of event scale: A measure of subjective stress. Psychosomatic Medicine, 41, 209-218.

Institute of Medicine. (2008). Treatment of posttraumatic stress disorder: An assessment of the evidence. Washington, DC: National Academies Press.

Keane, T., Fairbank, J., Caddell, J., Zimering, R., Taylor, K., \& Mora, C. (1989). Clinical evaluation of a measure to assess combat exposure. Psychological Assessment, 1, 53-55.

Kihlstrom, J. F. (2006). Trauma and memory revisited. In B. Uttl, N. Ohta, \& A. L. Siegenthaler (Eds.), Memory and emotion: Interdisciplinary perspectives (pp. 259-291). Malden, MA: Blackwell.

Kim, S. H., Schneider, S. M., Bevans, M., Kravitz, L., Mermier, C., Qualls, C., \& Burke, M. R. (2013). Symptom reduction with mindfulness-based stretching and deep breathing exercise: Randomized controlled clinical trial and efficacy. Journal of Clinical Endocrinology \& Metabolism, 98, 2984-2992.

Klein, E., Caspi, Y., \& Gil, S. (2003). The relation between memory for the traumatic event and PTSD. Evidence from Studies of Traumatic Brain Injury, 48, 28-33.

Kubany, E. S., Haynes, S. N., Leisen, M. B., Owens, J. A., Kaplan, A. S., Watson, S. B., \& Burns, K. (2000). Development and preliminary validation of a brief broad-spectrum measure of trauma exposure: The Traumatic Life Events Questionnaire. Psychological Assessment, 12, 210-224.

McNally, R. J. (2003a). Progress and controversy in the study of posttraumatic stress disorder. Annual Review of Psychology, 54, 229-252.

McNally, R. J. (2003b). Remembering trauma. Cambridge, MA: Harvard University Press.

Okuda, J., Fujii, T., Ohtake, H., Tsukiura, T., Tanji, K., Suzuki, K., . . Y Yamadori, A. (2003). Thinking of the future and past: The roles of the frontal pole and the medial temporal lobes. NeuroImage, 19, 1369-1380.

Ottaviani, R., \& Beck, A. T. (1987). Cognitive aspects of panic disorders. Journal of Anxiety Disorders, 1, 15-28.

Pearson, D. G., Deeprose, C., Wallace-Hadrill, S. M. A., Heyes, S. H., \& Holmes, E. A. (2013). Assessing mental imagery in clinical psychology: A review of imagery measures and a guiding framework. Clinical Psychological Review, 33, 1-23.

Report: More U.S. soldiers suffering from pre-traumatic stress disorder. (2006, November 15). Onion. Retrieved from 
http://www.theonion.com/articles/report-more-us-oldierssuffering-from-pretraumati,2088/

Rubin, D. C. (2014). Schema-driven construction of future autobiographical traumatic events: The future is more troubling than the past. Journal of Experimental Psychology: General, 143, 612-630.

Rubin, D. C., Berntsen, D., \& Bohni, M. K. (2008). A memorybased model of posttraumatic stress disorder: Evaluating basic assumptions underlying the PTSD diagnosis. Psychological Review, 115, 985-1011.

Schacter, D. L., \& Addis, D. R. (2007). The cognitive neuroscience of constructive memory: Remembering the past and imagining the future. Philosophical Transactions of the Royal Society. Series B: Biological Sciences, 362, 773-786.

Schacter, D. L., Addis, D. R., \& Buckner, R. L. (2007). Remembering the past to imagine the future: The prospective brain. Nature Reviews Neuroscience, 8, 657-661.

Spreng, R. N., \& Levine, B. (2006). The temporal distribution of past and future autobiographical events across the lifespan. Memory \& Cognition, 34, 1644-1651.

Suddendorf, T. (2010). Linking yesterday and tomorrow: Preschoolers' ability to report temporally displaced events. British Journal of Developmental Psychology, 28, 491-498.
Szpunar, K. K. (2010). Episodic future thought: An emerging concept. Perspectives on Psychological Science, 5, 142-162.

Szpunar, K. K., \& McDermott, K. B. (2008). Episodic future thought and its relation to remembering: Evidence from ratings of subjective experience. Consciousness and Cognition, 17, 330-334.

Szpunar, K. K., Watson, J. M., \& McDermott, K. B. (2007). Neural substrates of envisioning the future. Proceedings of the National Academy of Sciences USA, 104, 642-647.

Walker, R. W., Skowronski, J. J., \& Thompson, C. P. (2003). Life is pleasant-And memory helps to keep it that way. Review of General Psychology, 7, 203-210.

Weathers, F. W., Litz, B. T., Huska, J. A., \& Keane, T. M. (1994). The PTSD Checklist (PCL). Unpublished scale, available from the National Center for PTSD.

Weathers, F. W., Litz, B. T., Keane, T. M., Palmieri, P. A., Marx, B. P., \& Schnurr, P. P. (2013). The PTSD Checklist for DSM-5 $(P C L-5)$. Scale available from the National Center for PTSD, www.ptsd.va.gov

Williams, J. M. G., Ellis, N. C., Tyers, C., Healy, H., Rose, G., \& MacLeod, A. K. (1996). The specificity of autobiographical memory and imageability of the future. Memory $\varepsilon$ Cognition, 24, 116-125. 